\title{
3D Studio Production of Animated Actor Models
}

\author{
Adrian Hilton, Michael Kalkavouras and Gordon Collins \\ Centre for Vision, Speech and Signal Processing \\ University of Surrey, Guildford GU27XH, UK \\ a.hilton,m.kalkavouras,g.collins@ surrey.ac.uk
}

\begin{abstract}
This paper presents a framework for construction of detailed animated models of an actors shape and appearance from multiple view images. Multiple views of an actor are captured in a studio with controlled illumination and background. An initial low-resolution approximation of the persons shape is reconstructed by deformation of a generic humanoid model to fit the visual-hull using shape constrained optimisation to preserve the surface parameterisation for animation. Stereo reconstruction with multiple view constraints is then used to reconstruct the detailed surface shape. High-resolution shape detail from stereo is represented in a structured format for animation by displacement mapping from the low-resolution model surface. A novel integration algorithm using displacement maps is introduced to combine overlapping stereo surface measurements from multiple views into a single displacement map representation of the high-resolution surface detail. Results of 3D actor modelling in a 14 camera studio demonstrate improved representation of detailed surface shape such as creases in clothing compared to previous model fitting approaches. Actor models can be animated and rendered from arbitrary views under different illumination to produce free-viewpoint video sequences. The proposed framework enables rapid transformation of captured multiple view images into a structured representation suitable for realistic animation.
\end{abstract}

\section{Introduction}

Realistic representation of real people remains a primary goal of computer graphics research. Model construction in a structured format is a major bottleneck in the wide-spread use of shape capture for computer animation. Currently manual techniques are widely used in film production to build models of people suitable for realistic animation. 
Previous research has introduced the use of active 3D measurement technologies or multiple camera studios to capture the shape and appearance of a moving person $[11,18,30,17,4]$. Shape-from-silhouette and multiple view stereo are used to reconstruct sequences of 3D shape and appearance and render them from novel viewpoints. However, the resulting sequences are unstructured and do not allow modification of the actors movement.

Recent research $[3,25]$ has introduced model fitting techniques for automatic reconstruction of structured animated models from multiple view image sequences. Structured models allow modification of a persons movement in a standard animation pipeline and provide an efficient representation of a persons shape, appearance and motion. These approaches fit a generic humanoid model to the multiple view images using shape-from-silhouette[3] and model-based stereo refinement [25]. However, due to the fitting of a generic model to the captured data these approach do not accurately reproduce the fine detail of surface shape such as creases in clothing. There are two problems common to previous model fitting approaches: the visual-hull from multiple view silhouettes only provides a coarse representation of a persons shape without concavities; and fitting a generic (polygonal) model is limited to the degrees-of-freedom of the representation resulting in loss of fine surface detail.

Displacement mapping techniques have previously been used to represent fine surface detail for reconstruction of structured animated models from high-resolution range images of objects $[14,1,22,24]$. In this paper we introduce novel displacement mapping techniques for representation of surface detail reconstructed using passive stereo. Stereo reconstruction results in relatively noisy estimates of surface shape requiring robust methods for integration of overlapping surface measurements. Multiple view stereo and displacement mapping techniques are presented which allow detailed modelling of a persons shape in a structured form suitable for animation.

Section 2 presents a review of previous research addressing modelling people from multiple view images. The framework developed for reconstruction of detailed animated actor models is presented in section 3 together with novel algorithms for stereo reconstruction, displacement map stereo integration and representation of high-resolution surface detail. Results and evaluation of model reconstruction in a multiple camera studio environment are presented in section 4.

\section{Previous Work}

Advances in active sensor technology together with research in computer vision and graphics has resulted in systems for capturing surface models of complex 3D objects, people and internal environments $[5,7,16]$. These approaches capture 
accurate and realistic 3D models of complete objects with a level-of-detail not possible with previous manual techniques. However, such techniques result in object models which are represented as unstructured polygonal meshes consisting of millions of polygons. Conversion of such models to a structured form suitable for animation require labour intensive manual remeshing. Structuring captured data in a form suitable for animation is a challenging problem which has received only limited attention for character models [14, 15, 27] and people [9, 24]. Allen et al. [1] have recently used captured models of naked people in multiple static poses to animate a persons skin deformation during movement. However, active sensors are currently limited to the acquisition of static objects.

Research in computer vision has investigated passive methods for reconstructing models of people and their movement from multiple view images. Kanade et al.[11] demonstrated the first multiple camera studio to capture a moving persons 3D shape and appearance. This was used for 'Virtualised Reality' production of dynamic events with arbitrary camera viewpoint, illumination and background scene. Subsequent research [18, 30, 17, 4] has used shape-from-silhouette and multiple-view stereo techniques to reconstruct sequences of 3D people. These approaches produce an unstructured sequence of 3D surfaces, which do not support modification of the movement or efficient representation for animation. In addition, both multiple view stereo and shape-from-silhouette fail to accurately reconstruct the detailed surface shape due to visual ambiguities resulting in a loss of visual fidelity compared to the captured images [25].

Terzopoulos [28] introduced the reconstruction of structured 'functional models' instrumented for facial animation from captured 3D face shape of real people. This approach was applied to single camera reconstruction of animated models of people by fitting a generic humanoid model to silhouette images [6]. Recent research [3, 25] has addressed the problem of reconstructing structured models of people from multiple simultaneous camera views. These approaches fit a generic humanoid animation model to silhouette and stereo reconstruction from multiple camera views of a person in an arbitrary pose resulting in realistic models. Carranza et al.[3] automatically estimated the persons pose and fitted a polygonal humanoid model to the visual-hull reconstructed from multiple view image silhouettes. The quality of reconstructed texture mapped models is limited by the visual-hull which only reconstructs an approximate, locally convex, representation of the surface resulting in coarse geometry and visual artefacts due to incorrect alignment of overlapping images in texture map generation. Stereo surface has been used to improve reconstruction of surface detail [8, 20, 25]. Plaenkers and Fua [20] used implicit surface models to simultaneously reconstruct shape and movement from stereo point clouds. Results demonstrated reconstruction of complex movements with self-occlusion. Shape reconstruction is limited to a smooth surface due to the use of an implicit 
surface representation based on ellipsoidal volumetric primitives (meta-balls). Model-based reconstruction using shape constrained fitting [25] overcomes the limitations of shape-from-silhouette and stereo for reconstruction in the presence of visual ambiguities such as stereo correspondence in uniform surface regions. However, due to the fitting of a generic model with prior assumptions of local surface shape the reconstructed model may not accurately represent fine surface detail.

In this paper we build on previous approaches for model-based reconstruction of people [3, 25] by introducing techniques to reconstruct and represent additional high-resolution surface detail which is not represented in surface fitting. This approach combines model-based reconstruction of structured representations in the presence of visual ambiguities with accurate representation of surface detail which is not present in the generic model such as creases in clothing or hair.

\section{Reconstruction of Animated Actor Models}

This section presents the algorithms developed to reconstruct animated models of people from multiple view images. Section 3.1 presents an overview of the framework for model reconstruction. Subsequent sections present novel constributions of the constrained shape fitting, multiple view stereo, displacement map integration and representation algorithms.

\subsection{Model Reconstruction from Multiple Views}

The pipeline for reconstructing animated models from multiple view images is illustrated in Figure 1. The output from the system is a layered representation of the captured data which is structured in a form suitable for realistic and efficient animation. The representation consists of three layers: articulated skeleton, control model and displacement/texture map. The skeleton and control model provide a generic structure for animation with the control model surface non-rigidly deformed by animation of the skeleton. A displacement map is then used to represent the captured surface detail by mapping the stereo data onto the control model surface. This work introduces robust methods for displacement map representation of surface measurements from multiple passive stereo images with a relatively high noise level compared to previous methods applied to active range sensors. Texture mapping represents the detailed surface appearance reconstructed from multiple view images. This layered representation enables animation of the high-resolution captured surface detail based on deformation of the underlying control model.

The novel reconstruction approach combines narrow baseline stereo to estimate the detailed surface shape with wide baseline multiple view constraints for robust reconstruction. Novel displacement mapping algorithms are introduced to integrate 
estimates of surface shape from multiple stereo pairs and represent the surface detail. The challenge is to establish a mapping between the generic model and captured data with the correct correspondence for non-rigid deformation. Reconstruction of a layered representation from multiple view stereo data comprises five stages:

1. Model Registration: Initially the generic control model is manually posed for approximate alignment with the capture data. Joint centres are approximately aligned with the observed pose and constrained optimisation used to estimate the kinematic structure (limb-lengths) and pose. Anthropometric constraints preserve the skeletal symmetry of limblengths during fitting.

2. Shape Fitting: Shape constrained surface fitting is used to deform the control model surface to approximate the visualhull reconstructed from multiple view silhouettes [25]. Shape constraints preserve the control model parameterisation required for animation.

3. Stereo Reconstruction: Narrow-baseline stereo is used to reconstruct measurements of a surface shape from each adjacent camera pair. Wide baseline multiple view constraints are used to dynamically define the disparity search range, adapt the correlation window size and remove outliers from the stereo correspondence estimates. Stereo provides dense reconstruction of the detailed surface shape.

4. Displacement Mapping: Displacement mapping is used to integrate and represent the high-resolution estimates of surface shape from multiple view stereo. Normal-volume mapping [27] is used to establish a continuous correspondence between the generic model surface and each set of stereo data. Novel methods are introduced for integration of overlapping estimates of surface shape from stereo to reduce noise and reconstruct a single high-resolution surface model.

5. Texture Mapping: Multiple view stereo reconstructs a surface which aligns surface appearance between views. An integrated texture map image is reconstructed by combining the aligned images between multiple overlapping views. A multi-resolution Gaussian pyramid is used to combine overlapping images and fill texture holes in surface regions which are not visible from the captured views.

Steps 3 and 4 are new to the approach presented in this paper compared to previous model-based reconstruction algorithms $[3,25]$. This framework results in a layered representation of the person with the generic model structure and a common 
image-based representation of the high-resolution surface shape and appearance. The resulting model can be animated and rendered for production of image sequences with novel movement. Figure 2(a-d) presents an example of the reconstruction stages from the posed generic model(a), shape constrained fitting of the generic model (b), displacement mapping of highresolution surface detail from muliple view stereo (c) and final texture mapped model (d). It should be noted that there is significantly more geometric detail such as clothing folds in Figure 2(c) compared to the generic model fitting to silhouette and stereo data Figure 2(b).

\subsection{Shape Constrained Fitting}

Once the generic model is posed to match the 3D data set the next stage is to deform the shape of the generic control model so that it closely conforms to the 3D surface [25]. A requirement for natural animation of the conformed control model is that the mesh topology and vertex parameterisation does not change during conformance. A shape constrained deformable model is used to preserve the prior parameterisation of the control model while fitting to the 3D data set. The novelty of this approach lies in the formulation of a unique parameterisation for arbitrary triangular meshes which is used as the internal energy in mesh deformation.

The deformable surface model $\vec{x}$ minimises the energy function $E(\vec{x})$ incorporating the potential energy from data fitting $P(\vec{x})$, and the internal energy from the shape of the model $S(\vec{x})$.

$$
E(\vec{x})=P(\vec{x})+S(\vec{x})
$$

In previous work internal energy terms have been derived based on treating the surface as a membrane or thin-plate material under tension[28]. This yields the surface with minimum area or distortion that fits to the data. However, the thin-plate and membrane energy do not preserve the surface parameterisation which is essential for animation.

For an arbitrary triangular mesh a vertex position is not well defined in relation to the vertex neighbourhood. With an irregular number of vertices in the 1-neighbourhood it is not possible to obtain a consistent definition of a local frame to describe the position of the central vertex. We therefore consider a triangle face-based scheme as used by Kobbelt et. al. [13]. The vertex positions of a triangle face can be defined by the barycentric coordinates and height offset in the local frame of the vertices on the faces edge-connected to the central face, the vertices surrounding the central triangle.

The position of a mesh vertex is therefore constrained by the local position in the triangle face frames in the 1-neighbourhood 
of the vertex, leading to a 2-neighbourhood support structure for a vertex position.

We define the internal energy of a shape constrained model as the integral across the surface of the deviation in the local shape from the generic shape defined in each face based frame [26]. This is given by the summation of the error at the mesh vertices, $\vec{x}_{i}$, preserving the local parameterisation and shape in the vertex positions. Equation 2 defines the internal energy where $\left(\alpha_{i f}^{0}, \beta_{i f}^{0}, h_{i f}^{0}\right)$ are the default barycentric coordinates $(\alpha, \beta)$ and height offset $h$ in the $f^{t h}$ face based frame for the $i^{t h}$ vertex with valence $N_{i}$.

$$
S(\vec{x})=\sum_{i} \sum_{f} \frac{\left\|\vec{x}_{i}-\vec{x}\left(\alpha_{i f}^{0}, \beta_{i f}^{0}, h_{i f}^{0}\right)\right\|^{2}}{N_{i}}
$$

This constraint preserves the local shape of the generic humanoid model during fitting to ensure that the parameterisation of the control model $M^{L}$ for animation is preserved. For fitting to the visual-hull the external energy of equation $1 P(\vec{x})$ is derived by summing a data fit error $e(\vec{x})$ across the model surface $\vec{x}(u, v)$. We define the error metric in fitting the data as the least-squared error between the model and the 3D data set. The potential energy function is given by Equation 3 where $\vec{x}_{i}$ spans the set of $I$ model vertices and $\vec{y}_{j}$ spans the set of $J 3 \mathrm{D}$ data points.

$$
P(\vec{x})=\sum_{i} \sum_{j} m_{i j}\left\|\vec{y}_{j}-\vec{x}_{i}\right\|^{2}
$$

Fitting to the visual-hull is performed by an iterative gradient descent solution to minimise the energy function equation 1. Further details of the fitting procedure are presented in [25]. Figure 2(a),(b) show an example of the control model surface before and after shape constrained fitting to visual-hull. This process preserves the generic control model animation structure and mesh parameterisation allowing the resulting model to be animated.

\subsection{Stereo Surface Reconstruction}

Stereo produces detailed surface reconstruction in regions of non-uniform appearance, and noisy or erroneous measurements in regions of uniform appearance. In this work we enhance existing stereo algorithms [19, 12] to improve reconstruction accuracy and reduce outliers for multiple camera capture in a controlled studio environment. Three novel constraints for multiple view stereo are introduced: elimination of correspondences outside the visual-hull; a dynamic disparity range for correspondence inside the visual-hull; and neighbourhood consistency for ambiguous correspondences. An adaptive correlation window size is also used to avoid ambiguities in regions close to the silhouette boundary. The enhanced stereo 
algorithm reduces the number of false correspondences which would result in outliers or noise in conventional pair wise stereo algorithms.

Dense stereo correspondence between each pair of adjacent cameras is estimated as follows:

1. Rectification: Images are rectified in order to align the epipolar lines horizontally between camera views.

2. Dynamic disparity range: The disparity range to search for correspondence between images is calculated dynamically for each pixel. The disparity range is constrained to lie inside the visual hull with a maximum distance $d_{\max }$ from the visual hull surface. The ray corresponding to a pixel in one image is intersected with the visual-hull to evaluate the segment of the ray inside the visual hull with distance less than $d_{\max }$. The corresponding section of the epi-polar line in the other image is then used to constrain the correspondence search. This procedure is performed in both directions to ensure consistency of stereo correspondence. A value $d_{\max }=0.1 \mathrm{~m}$ has been used throughout this work, which corresponds to the maximum expected deviation between the visual-hull and true surface together with calibration error between views of $\approx 0.01 \mathrm{~m} \equiv 1 \mathrm{pixel}$. The resulting disparity search range along the epi-polar line is approximately 10 pixels. Dynamic disparity range reduces the number of incorrect stereo matches and improves computational efficiency.

3. Disparity search: Normalised cross-correlation is used to measure correspondence between an $n \times m$ pixel window in each image by searching along the epi-polar line within the disparity range. Adaptive matching is used to reduce the window size in regions close to the silhouette boundary whilst maintaining a larger window size in internal regions for accurate localisation. All correlation peaks above a threshold $t_{c}$ are identified and stored. In this paper a window sizes of $11 \times 11$ is used in interior regions and $5 \times 5$ in regions near the silhouette border. Windows which fall outside the silhouette boundary are excluded. A correlation threshold $t_{c}=0.7$ has experimentally been found to give a good compromise between identifying the correct and spurious correlation peaks. Note: pixels with multiple peaks are disambiguated in subsequent processing.

4. Neighbourhood consistency: A two-pass algorithm is introduced to estimate the correct disparity for each pixel. In the first pass only pixels with a single disparity peak above the correlation threshold $t_{c}$ are accepted. The second pass uses a median filter to identify the median neighbourhood disparity peak for adjacent pixels with an unambiguous single disparity value. The correct peak for a pixel with multiple peaks is constrained to lie within $\pm t_{d} p i x e l s$ of the median neighbourhood disparity. Throughout this work $t_{d}=2$ pixels is used, this is the minimum distance between 
adjacent peaks and imposes a strict threshold to robustly eliminate incorrect peaks. Neighbourhood consistency results in a dense disparity map with holes in ambiguous surface regions due to low correlation peak or ambiguity in local appearance.

5. Noise removal: A second median filter with a window size equal to the correlation matching window size is applied to all disparity estimates to remove remaining outliers and reduce noise. Any disparity estimate that is outside $\pm t_{d} p i x e l s$ of the median disparity for the corresponding correlation window is removed as noise. This reduces correspondence noise in the dense disparity map by ensuring neighbourhood consistency for smooth surfaces. The assumption of smooth surfaces is valid in the reconstruction of stereo disparity maps for people where the visual-hull constraint eliminates surface discontinuities.

6. Sub-pixels disparity estimation: A quadratic function is fitted to the correspondence estimates over a 5 pixel neighbourhood.

The stereo algorithm results in a dense disparity map with relatively few outliers due to incorrect correspondence or noise. Due to the redundancy of overlapping disparity maps from multiple views elimination of outliers is preferable to recovering a surface estimate at every pixel. Figure 3 presents results of the stereo reconstruction for one camera pair. Dense stereo is reconstructed for a large proportion of the surface but holes also occur due to the robust outlier removal using the multiple view constraints. Figures 3(c) and (d) show the stereo reconstruction of high-resolution surface detail such as ridges in the clothing. This high-resolution surface detail is not present in the model resulting from the multiple view model fitting [25]. Integration of reconstruction from multiple stereo views is used to remove holes and obtain high-resolution data for the entire surface.

\subsection{Integration of Multiple View Stereo}

In this section we introduce a robust method to integrate estimates of surface shape from multiple stereo pairs into a single high-resolution surface. Previous algorithms for integration of multiple view range images $[5,7,21]$ assume relatively accurate surface measurements from active 3D sensors using projected structured light. Stereo estimates of surface geometry for people in normal clothing are relatively noisy due to the absence of local variations in surface appearance required for accurate correspondence localisation. Therefore, a robust approach to integration of surface measurements is required. 
In this paper we introduce a novel approach to integration of surface shape estimates from stereo using displacement maps. Initially a displacement map image represented is reconstructed for the surface estimates from each stereo pair. Robust integration of individual displacement map images is then performed to obtain a unified displacement map image representation of the high-resolution surface detail.

Displacement mapping using the normal-volume was previously introduced [27] to allow representation and animation of a high-resolution polygonal surface model, $M^{H}$, as displacements from a low-resolution polygonal animation control model, $M^{L}$. The normal-volume for triangles on a polygonal mesh, $M^{L}$, defines a continuous spatial mapping between the surface of the low-resolution model and the volume occupied by the high-resolution model, $M^{H}$. Previous work [27, 24] used this approach to efficiently represent high-resolution surface measurements captured using active 3D sensors such as laser scanners. It was also shown that this parameterisation could be used to seamlessly animate the high-resolution model based on deformation of the underlying control model.

In this work we use the normal-volume displacement mapping technique to represent and integrate the relatively noisy surface measurements from multiple view stereo. Displacement map representation of individual stereo shape estimates allows the correspondence between multiple noisy sets of overlapping surface measurements to be established. This overcomes limitations of previous implicit surface $[5,7]$ and mesh-based nearest-point $[23,29]$ range image integration algorithms in the presence of significant measurement noise.

The multiple view stereo integration algorithm is performed in the following steps:

1. Stereo Reconstruction: Stereo reconstruction of dense disparity image $I_{i j}$ between adjacent camera views $i$ and $j$ is performed using the algorithm introduced in section 3.3.

2. Constrained Triangulation: For each pixel $I_{i j}(r, s)$ in the disparity image, for which a valid disparity has been estimated, we estimate the corresponding 3D surface position $\vec{x}(r, s)$. A high-resolution triangulated mesh $M_{i j}^{H}$ is then constructed by constrained triangulation [7] of adjacent surface position estimates $\vec{x}(r, s), \vec{x}(r+1, s), \vec{x}(r, s+$ 1), $\vec{x}(r+1, s+1)$. A triangle is added to the mesh if the Euclidean distance between adjacent surface measurements $|x(r, s)-x(p, q)|<d_{t}$, where $d_{t}=3 \delta x$ and $\delta x$ is the surface sampling resolution. For a capture volume of $2 m^{3}$ with image resolution $720 \times 576$ we have $\delta x \approx 0.01 m$ which is used throughout this work. Constrained triangulation has been used in previous range image integration algorithms to construct a mesh which approximates the local surface 
topology [5, 7]. This results in a mesh $M_{i j}^{H}$ which approximates the local surface geometry but does not connect across step discontinuities which occur at occlusion boundaries.

3. Displacement Map Representation: Normal-volume displacement mapping [27] is used to obtain a displacement image representation $D_{i j}(u, v)$ of the high-resolution stereo mesh $M_{i j}^{H}$ mapped onto the low-resolution surface $M^{L}$ of the generic model fitted to the visual-hull in step 2 of the multiple view reconstruction algorithm. Two-dimensional texture image coordinates $\vec{u}=(u, v)$ for each vertex $\vec{v}^{L}$ of the low-resolution model $M^{L}$ are used to map the normalvolume displacements to a two-dimensional displacement image $D_{i j}(u, v)$ in a process analogous to texture mapping. Steps 1-3 result in a displacement map image $D_{i j}(u, v)$ for each stereo pair in a common 2D coordinate frame, as shown in Figure 4(a-c).

4. Displacement Map Integration: Individual displacement map images $D_{i j}(u, v)$ are integrated into a single displacement map $D(u, v)$ using a robust filtering approach. For a pixel $(u, v)$ in the integrated displacement map image $D$ we compute the average neighbourhood displacement $d_{\text {ave }}(u, v)$ using an $n \times m$ window: $d_{\text {ave }}(u, v)=$ $\frac{1}{n m} \sum_{p=u \pm \frac{n}{2}, q=v \pm \frac{m}{2}} D(p, q)$. We then obtain the displacement map with a value closest to the average neighbourhood displacement for all individual displacement maps $D_{i j}$ with valid displacement values at pixel $(u, v)$ : $d(u, v)=\min \left|d_{i j}(u, v)-d_{a v g}(u, v)\right|$. If there are no valid neighbourhood displacements then we select the smallest displacement value which lies closest to the visual-hull. Throughout this work a $5 \times 5$ window is used to evaluate the average neighbourhood displacement at a given pixel. The result is a single displacement map image $D(u, v)$ which represents the integrated high-resolution surface detail from multiple stereo pairs, as shown in Figure 4(d).

The integration algorithm overcomes a number of limitations of previous approaches to allow integration of relatively noisy 3D surface measurements from passive stereo. Specifically the integration algorithm avoids: averaging of overlapping surface measurements which may reduce reconstructed surface detail due to misalignment; step discontinuities which occur at transitions between overlapping sets of surface measurements if no averaging is used; and inclusion of outliers which are inconsistent with the reconstructed surface in a local neighbourhood. Previous range image integration algorithms $[5,7]$ use surface visibility to combine measurements based on either a weighted average or best-view criteria. In the case of relatively inaccurate stereo measurements it has been found that averaging results in a loss of surface detail. Likewise use of a best-view criteria results in visible step discontinuities at the transition between estimates of surface shape from different stereo pairs. 
The closest value approach introduced in this work overcomes these problems, resulting in reconstruction of a continuous high-resolution surface representation with preservation of surface detail from individual stereo views.

Results from our displacement map integration algorithm can be seen in Figure 4. Three displacement map images for individual stereo pairs (right,middle and left) are shown in Figures $4(\mathrm{a}-\mathrm{c})$. Note the holes in individual displacement maps which are shown in the same uniform grey as the background indicating zero displacement. Figure 4(d) shows the integrated displacement map image with holes filled from the data in individual views and preservation of the detailed high-resolution structure from individual stereo pairs. Displacement map images are coded in the range \pm 0.02 indicating the difference between the low-resolution control model and the detailed surface reconstructed using stereo.

\subsection{Texture Map Generation}

The final stage in model reconstruction is integration of the multiple view images into a single texture image [25]. Stereo reconstruction between adjacent camera views gives good estimates of correspondence for surface regions with local variation in appearance. This correspondence is used to align the multiple view images and resample a single texture image for the model surface. Accurate correspondence reduces misalignment in the texture map between visible features which simplifies the generation of a single texture map image. Texture integration is performed in the following steps:

1. Texture mapping individual images: A texture map image $T_{i j}$ is computed for each stereo pair using the corresponding displacement/texture map coordinates $D_{i j}$ for the estimated surface shape mapped onto the low-resolution model $M^{L}$. The captured image $I_{i}(r, s)$ is then resampled into the texture image $T_{i j}(u, v)$ using bilinear interpolation for each pixel $(r, s)$ with a valid stereo correspondence. For invalid pixels with no stereo match, which occur in regions of uniform appearance, a zero displacement value is assumed and texture resampling is performed to avoid holes in the resulting texture map. This results in a set of overlapping texture map images $T_{i j}(u, v)$ in the two-dimensional texture image space $(u, v)$ defined a priori for the low-resolution model $M^{L}$.

2. Texture map integration: Integration of texture map images $T_{i j}$ into a single texture map is performed using a bestview criteria. Initially for each pixel $(u, v)$ data from the best-view $T_{p q}(u, v)$ with a valid displacement map estimate $D_{p q}(u, v)$ are resampled into the integrated texture map image $T(u, v)$. The best-view criteria uses the angle between the camera view and the surface normal [25]. Any missing pixels are then filled in iteratively with the next best-view 
having a valid displacement map value. Finally, holes with no estimated displacement map are filled with the image data from the best-view based on the angle between the low-resolution model $M^{L}$ surface normal and the viewing direction. This process resamples the multiple view images $I_{I}(r, s)$ into a single texture map image $T(u, v)$. Surface regions which are not visible in any of the captured images due to self-occlusion will result in holes in the texture map.

3. Texture hole filling: A multi-resolution Gaussian image pyramid [2] is used to fill in any holes of the integrated texture map. Each image in the pyramid is convolved with a $5 \times 5$ Gaussian kernel, starting with the initial integrated texture image. The result is a low-pass filtered version of each image in which the resolution is reduced by half at each step. The missing texture pixels at each resolution are then filled starting at the penultimate resolution of the pyramid by bilinear interpolation of the pixel values from the proceeding lower resolution image.

In figure 5 we can see texture from different stereo pairs, an integrated texture map and the result of using a multi-resolution Gaussian image pyramid to fill in the holes. Two of the four derived texture maps are shown. The integrated texture map from all stereo views is shown in 5(c). The result of using a multi-resolution Gaussian image pyramid is shown in 5(d). This process results in a single integrated surface model which combines the stereo data from multiple views to represent highresolution surface detail, as illustrated in Figure 2(d). The resulting layered model can then be animated efficiently via the skeleton articulation structure and control model $M^{L}$. High-resolution surface detail is reconstructed from the displacement map $D$ based on the deformation of the control model surface.

\section{Results}

In this section we present our results of animated models generated using multiple view images captured in a studio environment.

\subsection{Multiple Camera Studio}

Two different studio setups have been used for model generation: an 8 camera configuration with narrow baseline stereo from the front and sides; and a 13 camera configuration with narrow baseline stereo from all sides. Both setups have a capture volume of approximately $2 \mathrm{~m}^{3}$ with the cameras placed at a distance of $4 \mathrm{~m}$ from the centre. All cameras are Sony DXC 9100P progressive scan 3CCD with a PAL image resolution of $720 \times 576$. 
In the first setup we have used a system of 8 cameras, with 5 cameras placed side by side with a baseline of $50 \mathrm{~cm}$ facing the person, 2 cameras on the side of the person and 1 above the person facing down. All of the cameras are used for extracting the visual hull, but only the five frontal cameras are used for stereo. A matlab chart based camera calibration toolbox is used giving an rms error of approximately 0.5 pixels.

The second setup consists of 13 cameras, with 4 cameras facing the front of the model and 4 facing the back, 2 cameras positioned on each side, and 1 above the person facing down. All 13 cameras are used to extract the visual hull and only the top camera is not used for stereo. Chart camera calibration is used to independently calibrate front and back cameras followed by registration of the estimated coordinate frames using the common calibration overhead and side cameras.

Stereo for a single pair takes around 2 minutes on a PentiumIII 900MHz machine for each camera pair, and a complete model with manual posing could be reconstructed in less than 2 hours.

\subsection{Shape Reconstruction}

Figure 6 shows results of three models of people generated with the 8 camera setup. The resulting models demonstrate the inclusion of high-resolution surface detail from the multiple view stereo. Comparison with the fitted low-resolution generic model used in previous model-based multi-view reconstruction [25] demonstrates significant improvement in the reconstruction of detailed surface geometry such as creases in the clothing. Reconstruction of significant features is visible above the level of the stereo noise as shown in Figure 3. The difference between the low-resolution model reconstruction [25] and the displacement mapped models reconstructed in this work is of the order $\pm 0.02 m$. Geometric artefacts remain in regions such as the hands and face where the stereo algorithm fails to reconstruct shape detail. The visual quality of the resulting texture mapped models compared is comparable to the captured images. Stereo reconstruction results in correct alignment of the surface detail in regions with significant local variation in appearance such as the collar line, patterns on the clothing and creases.

Figure 7 shows an example of a complete model using the multiple view stereo and displacement mapping to reconstruct high-resolution surface detail. Results demonstrate representation of detail such as creases in clothing which is a significant improvement over the fitted generic model. Figure 7 shows the displacement map image together with a histogram of the displacement map values in the range $\pm 0.02 \mathrm{~m}$ from the low-resolution model surface. As expected the average displacement from the low-resolution model is approximately zero which confirms a good fit to the date. The distribution indicates a 
bias towards negative displacements which is expected as the visual-hull used in the model fitting is an over-estimate of the surface shape. A significant proportion of the displacement values are at the extremities (greater than $0.01 \mathrm{~m}$ or less than $-0.01 \mathrm{~m}$ ) indicating that the displacement mapped model represents significant geometric structures not present in the low-resolution model. The texture mapped models contain some visible artefacts on the sides due to the texture integration algorithm resulting in blurred texture in regions which were not reconstructed in the captured stereo pairs. Overall the multiple view stereo reconstruction results in improved geometric accuracy and visual fidelity over previous model-based approaches $[3,25]$.

\subsection{Model Animation}

One of the advantages of using displacement maps is that we can easily animate the resulting model using commercial animation packages such as 3D Studio MAX with displacement and texture mapping to animate the high-resolution surface detail. Results of animating the model shown at Figure 7 are presented in Figure 8. The sequence presented demonstrates animation of the high-resolution surface detail such as creases in the clothing based on deformation of the underlying generic control model. In this example a simple skeleton based vertex weighting scheme is used to animate the control model surface resulting in thinning around the shoulder joint. A more sophisticated animation scheme could be used for animation production with displacement mapping used to represent and reconstruct surface detail during animation.

\section{Conclusions}

In this paper we have presented a framework for reconstruction of animated models of people from multiple view images captured in a studio environment. This paper extends previous model-based multiple view reconstruction of people $[3,25]$ to reconstruct and represent the detailed surface shape from multiple view stereo. Stereo reconstruction has been extended using multiple view constraints to achieve dense reconstruction of high-resolution surface detail. Robust constraints are introduced to eliminate outliers and reduce noise in the stereo reconstruction. A novel method using displacement mapping to represent and integration estimates of surface shape from multiple views has been presented. This approach allows integration of relatively noisy estimates of surface shape from stereo overcoming limitations of previous range image integration algorithms for noisy data $[5,7]$.

Results demonstrate the reconstruction of models of people which represent the detailed surface shape of clothing. Model- 
based stereo achieves reconstruction of the detailed surface shape such as creases in clothing. The size of the features is in the range $\pm 0.02 \mathrm{~m}$ representing significant geometric details when visualising the model. Currently the stereo reconstruction of smaller features detail such as fingers, face and hair is limited by the camera image resolution which is $\approx 0.01 \mathrm{~m}$. Displacement map integration and representation of multiple view stereo represents the high-resolution surface shape not present in the underlying model. This overcomes limitations of previous model-based [3, 25] and non-model based [10, 30, 4, 17] approaches to reconstructing and representing people from multiple camera views. Future research will investigate the use of displacement mapping techniques to reconstruct and represent the detailed high-resolution surface dynamics from multiple view stereo such as cloth deformation.

\section{Acknowledgements}

This research was supported by EU IST FW5 Project MELIES and EPSRC Platform Grant GR/S13576.

\section{References}

[1] B. Allen, B. Curless, and Z. Popovic. Articulated body deformation from range scan data. In Proc. ACM SIGGRAPH, pages 612—619, 2002.

[2] P.J. Burt and E.H. Adelson. A Multiresolution Spline with Application to Image Mosaics. ACM Trans.Graph., 2(4):217—236, 1983.

[3] J. Carranza, C. Theobalt, M. Magnor, and H.-.P. Seidel. Free-viewpoint video of human actors. In Proc. ACM SIGGRAPH, pages 565-577, 2003.

[4] G.K.M. Cheung, S. Baker, and T. Kanade. Visual Hull Alignment and Refinement Across Time: A 3D Reconstruction Algorithm Combining ShapeFrom-Silhouette with Stereo. In Conference on Computer Vision and Pattern Recognition, pages 375-382, 2003.

[5] B. Curless and M. Levoy. A Volumetric Method for Building Complex Models from Range Images. In ACM Computer Graphics Proceedings, SIGGRAPH, NewOrleans, USA, pages 303-312, 1996.

[6] A. Hilton, D. Beresford, T. Gentils, R. Smith, W. Sun, and J. Illingworth. Whole-body modelling of people from multi-view images to populate virtual worlds. Visual Computer: International Journal of Computer Graphics, 16(7):411-436, 2000. pdf.

[7] A. Hilton, A.J. Stoddart, J. Illingworth, and T. Windeatt. Implicit surface based geometric fusion. International Journal of Computer Vision and Image Understanding, Special Issue on CAD Based Vision, 69(3):273-291, March 1998. pdf.

[8] J. Isidoro and S. Sclaroff. Stochastic Refinement of the Visual Hull to Satisfy Photometric and Silhouette Consistency Constraints. In International Conf. on Computer Vision, pages 1335-1342, 2003.

[9] X. Ju and J.P. Siebert. Conformation from generic animatable models to 3d scanned data. In International conference of 3D Scanning, Paris.

[10] T. Kanade. Virtualized reality: putting reality into virtual reality. In 2nd International Workshop on Object Representation for Computer Vision ECCV, 1996. 
[11] T. Kanade and P. Rander. Virtualized reality: Constructing virtual worlds from real scenes. IEEE MultiMedia, 4(2):34—47, 1997.

[12] S.B. Kang and R. Szeliski. 3D Scene Recovery using Omnidirectional Multibaseline Stereo. In Conference on Computer Vision and Pattern Recognition, pages 364-372, 1996.

[13] L. Kobbelt, S. Campagna, J. Vorsatz, and H.P. Seidel. Interactive multi-resolution modeling on arbitrary meshes. In Proc. ACM SIGGRAPH, pages 105-114, 1998.

[14] V. Krishnamurthy and M. Levoy. Fitting smooth surfaces to dense polygon meshes. In In ACM Computer Graphics Proc. SIGGRAPH, NewOrleans, USA, 1996.

[15] A. Lee, H. Moreton, and H. Hoppe. Displaced subdivision surfaces. In Proc. ACM SIGGRAPH, pages 85—94, 2000.

[16] M. Levoy, K. Pulli, B. Curless, S. Rusinkiewicz, D. Koller, L. Periera, M. Ginzton, S. Anderson, J. Davis, J. Ginsberg, J. Shade, and D. Fulk. The Digital Michelangelo Project. In ACM Computer Graphics Proceedings, SIGGRAPH, pages 131-144, 2000.

[17] W. Matusik, C. Buehler, R. Raskar, and S. Gortler. Image-based visual hulls. In Proc. ACM SIGGRAPH, pages 369-374, 2000.

[18] S. Moezzi, L-C. Tai, and P. Gerard. Virtual view generation for 3d digital video. IEEE MultiMedia, 4(2):18—26, 1997.

[19] M. Okutomi and T. Kanade. A locally adaptive window for signal matching. International Journal of Computer Vision, 7(2):143-162, 1992.

[20] R Plankers and P. Fua. Articulated soft objects for video-based body modeling. IEEE International Conference on Computer Vision, pages 394-401, 2001.

[21] S. Rusinkiewicz, O. Hall-Holt, and M. Levoy. Real-time 3d model acquisition. In Proc. ACM SIGGRAPH, 2002.

[22] R. Smith, W. Sun, A. Hilton, and J. Illingworth. Layered animation using displacement maps. In IEEE International Conference on Computer Animation, pages 146-154, May 2000. pdf.

[23] M. Soucy and D. Laurendeau. A general surface approach to the integration of a set of range views. IEEE Trans. Pattern Analysis and Machine Intelligence, 14(4):344-358, 1995.

[24] J. Starck, G. Collins, R. Smith, A. Hilton, and J. Illingworth. Animated statues. Journal of Machine Vision Applications, 14(4):248-259, 2003. pdf.

[25] J. Starck and A. Hilton. Model-based multiple view reconstruction of people. In IEEE International Conference on Computer Vision, pages 915-922, 2003. pdf.

[26] J. Starck and A. Hilton. Reconstruction of animated models from images using constrained deformable surfaces. In 10th Conf. on Discrete Geometry for Computer Imagery. Vol.2301, Lecture Notes in Computer Science, Spriger-Verlag, pages 382-391, Bordeaux, France, April 2002. pdf.

[27] W. Sun, A. Hilton, R. Smith, and J.Illingworth. Layered animation of captured data. Visual Computer: International Journal of Computer Graphics, 17(8):457-474, 2001. pdf.

[28] D. Terzopoulos. From physics-based representation to functional modeling of highly complex objects. In NSF-ARPA Workshop on Object Representation in Computer Vision, pages 347-359. Springer Verlag, 1994.

[29] G. Turk and M. Levoy. Zippered Polygon Meshes from Range Images. In ACM Computer Graphics Proceedings, SIGGRAPH, Orlando, Florida, pages 311-318, 1994. 
[30] S. Vedula, S. Baker, and T. Kanade. Spatio-temporal view interpolation. Eurographics Workshop on Rendering, pages 1-11, 2002.

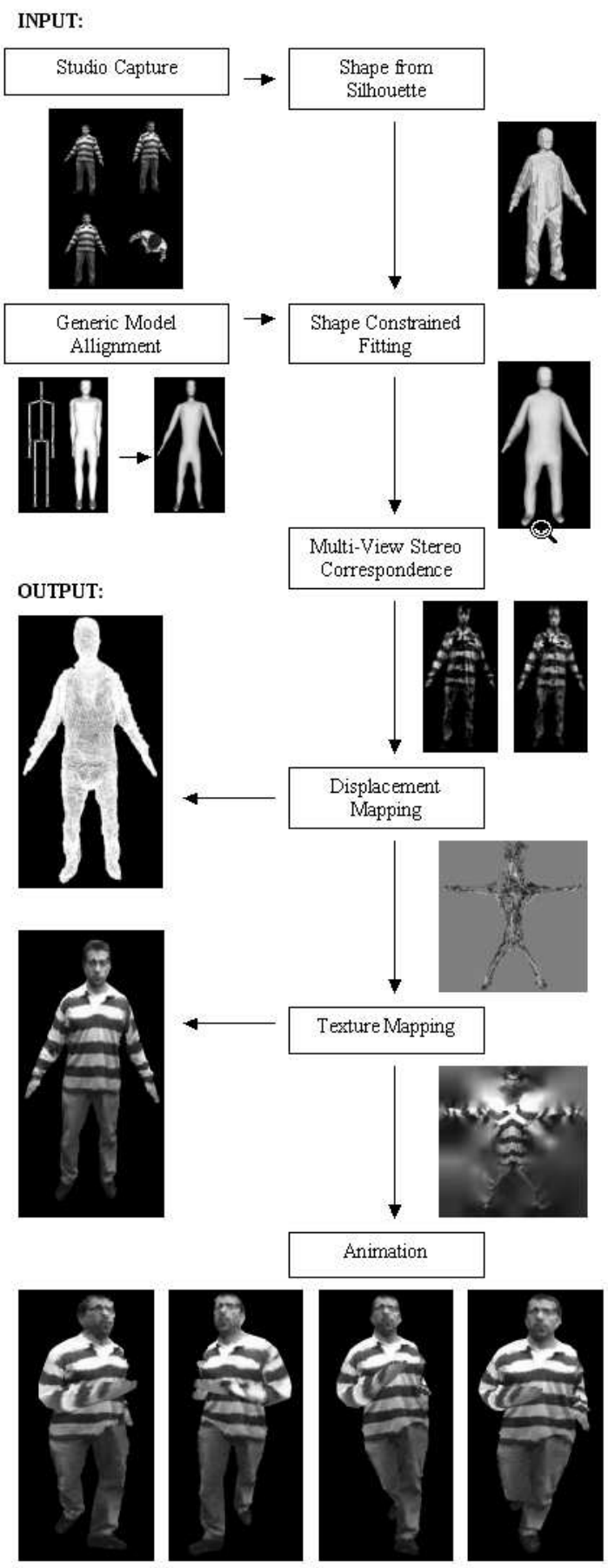

Figure 1. Overview of framework for producing animated actor models from multiple view studio capture 

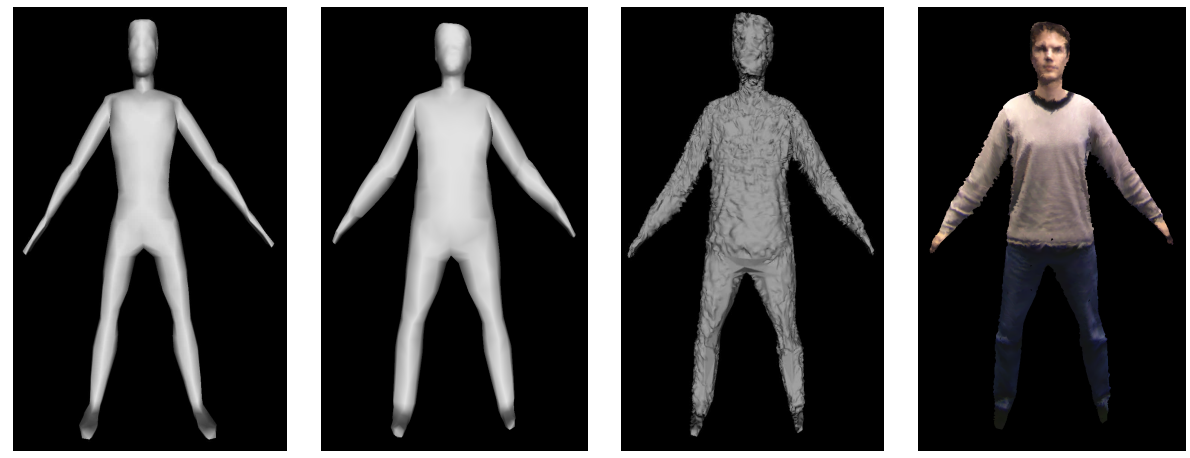

(a) Posed control model

(b) Control model fit to

(c)

High-resolution

(d) High-resolution

visual-hull

model

model with texture

Figure 2. Sequence of model reconstruction 

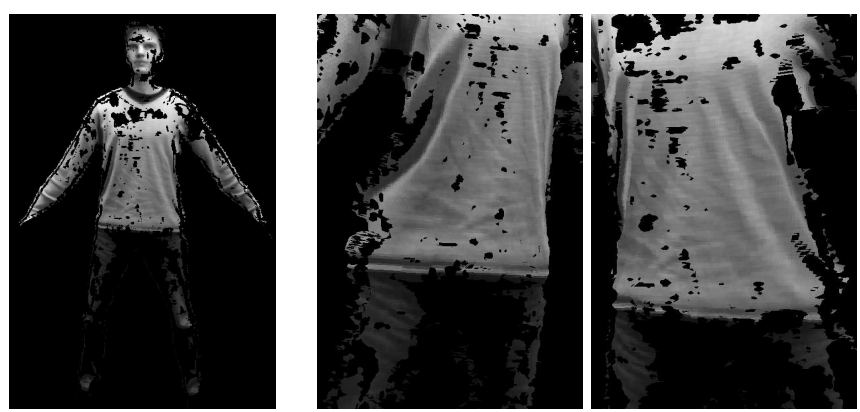

(a) Full-body

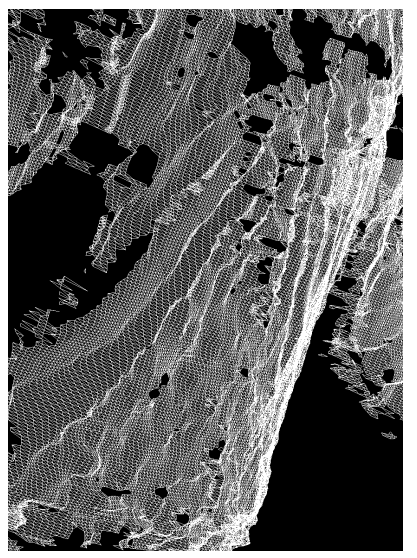

(b) Close-up torso

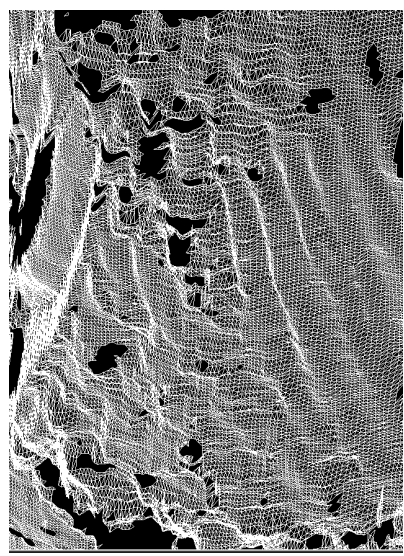

(c) Close-up with no texture (Wireframe)
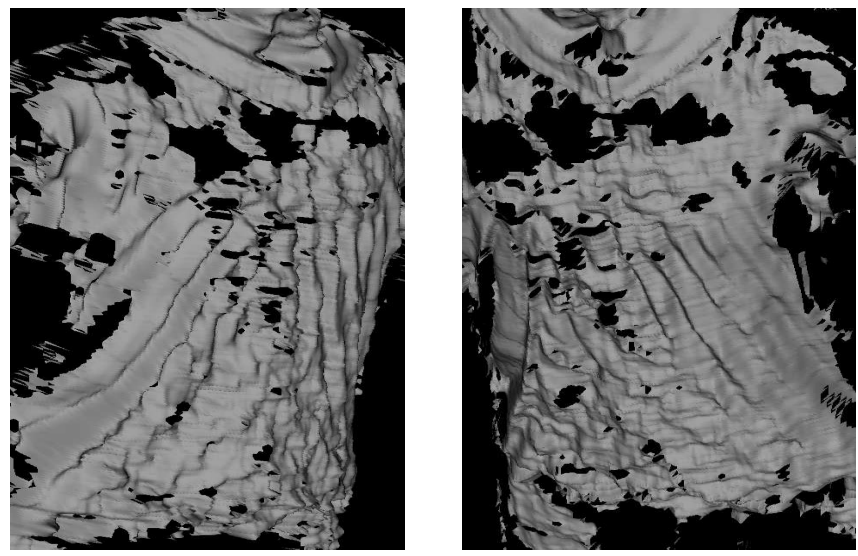

(d) Close-up with no texture (Shading)

Figure 3. Stereo results from one camera pair. Note the reconstruction of detailed creases in the clothing shown in (c) and (d). 


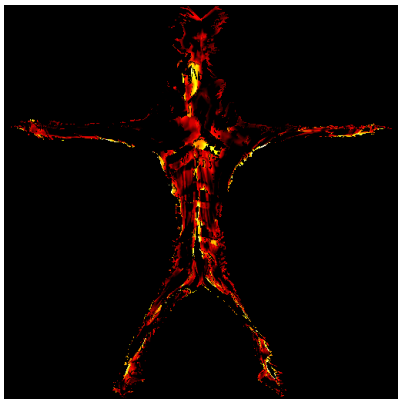

(a) Right View

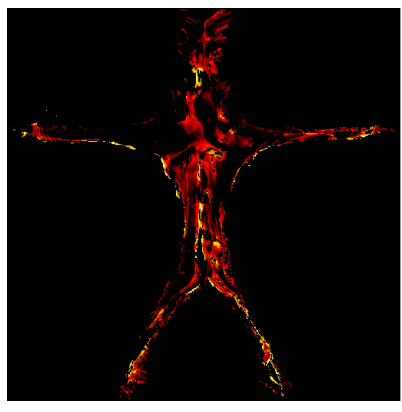

(b) Middle View

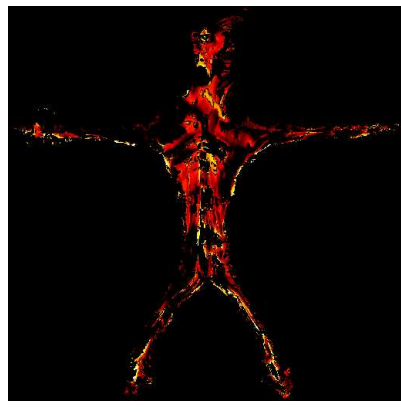

(c) Left View

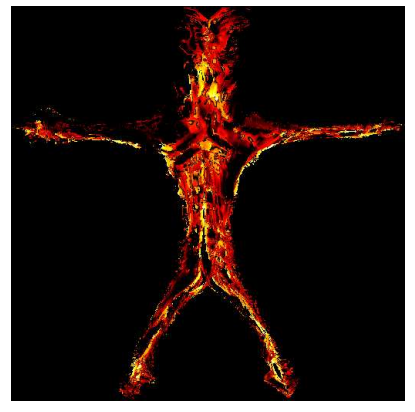

(d) Integrated

Figure 4. Displacement map images for multiple stereo views and integrated displacement map image. Displacements are colour coded from black $(-0.02 \mathrm{~m})$ to white $(0.02 \mathrm{~m})$ relative to the control model surface (see Figure 7(d)), the uniform black background colour indicates zero displacement.

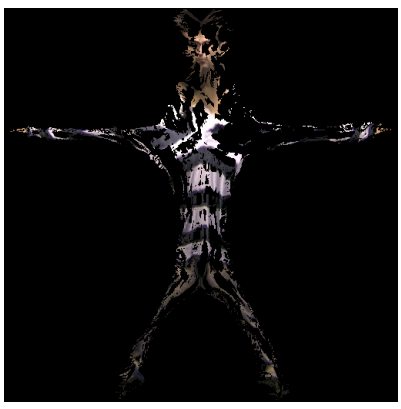

(a) Right View

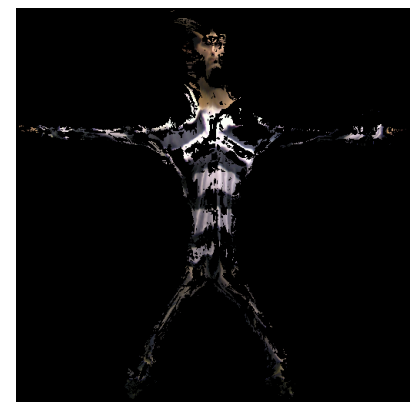

(b) Left View

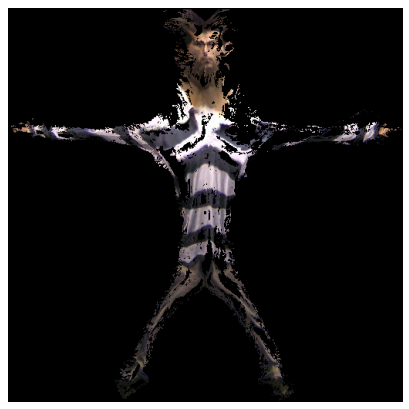

(c) Integrated Texture

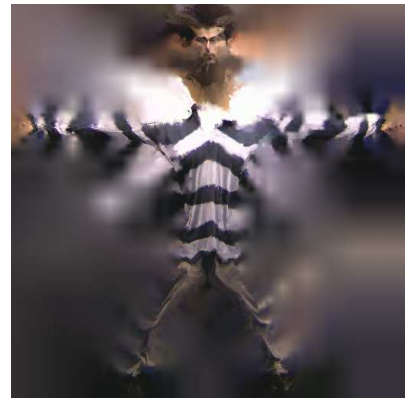

(d) Blended Texture Map

Figure 5. Texture blending of stereo views 

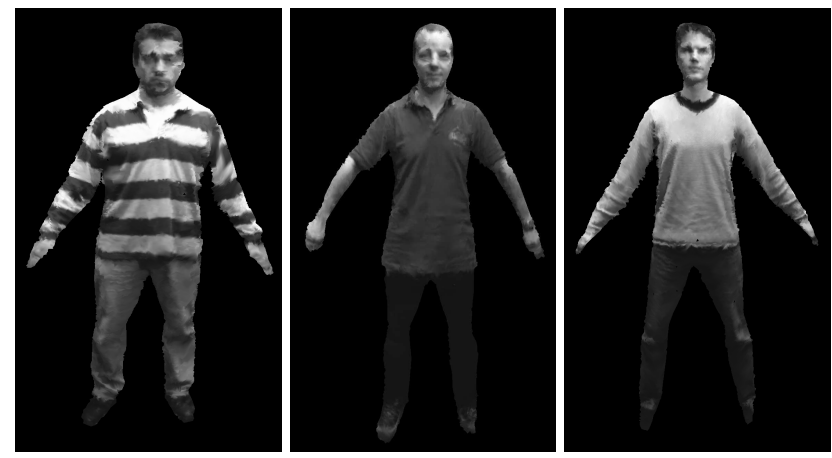

(a) Reconstructed models with texture
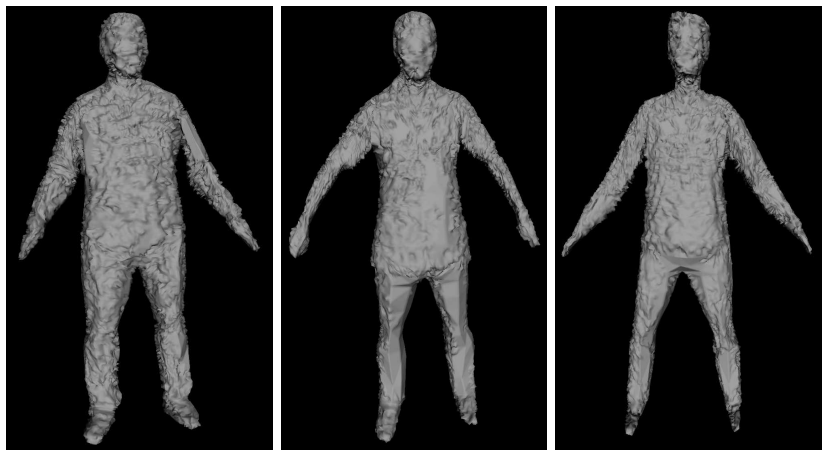

(b) Reconstructed models shape (shading)

Figure 6. Reconstruction from Multiple Front Views 

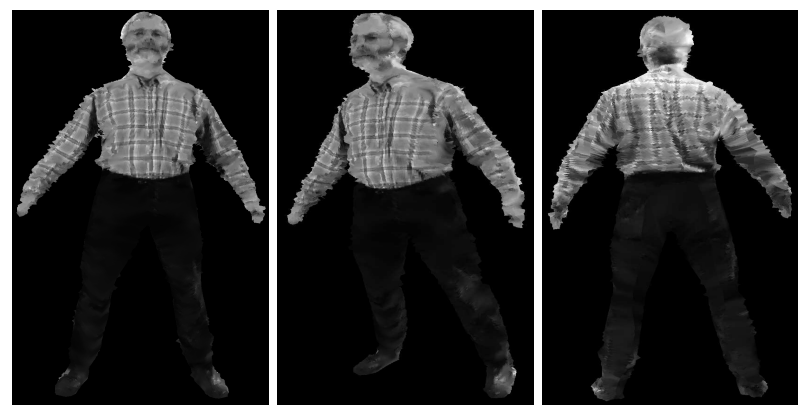

(a) Multiple texture mapped views
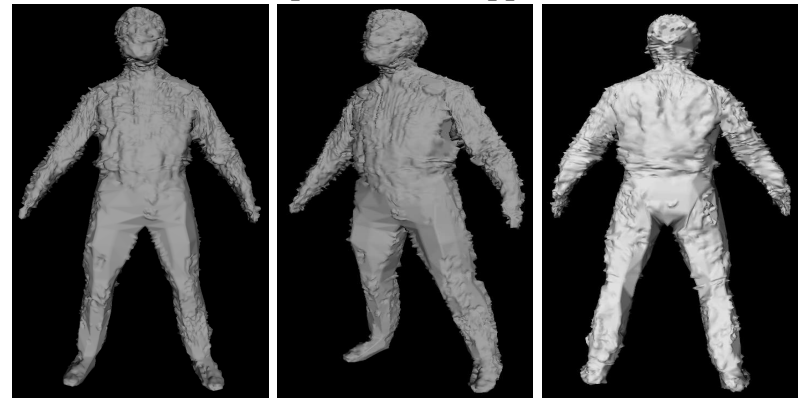

(b) Multiple flat shaded views

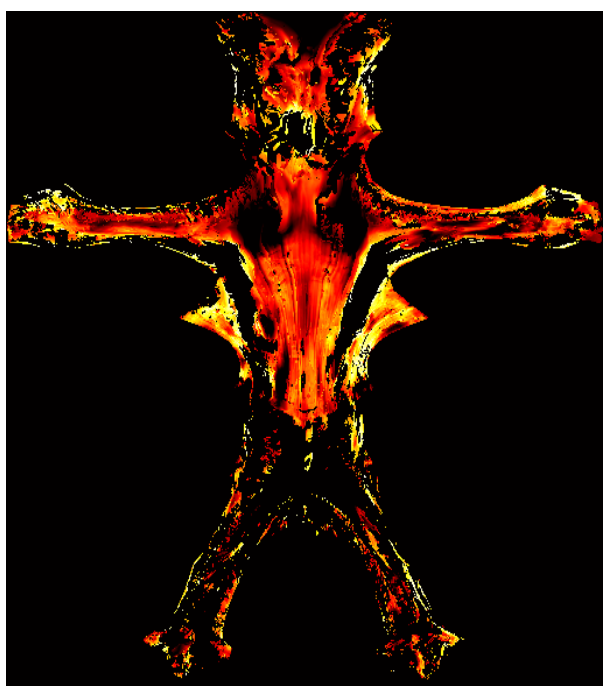

(c) Colour mapped displacement map image

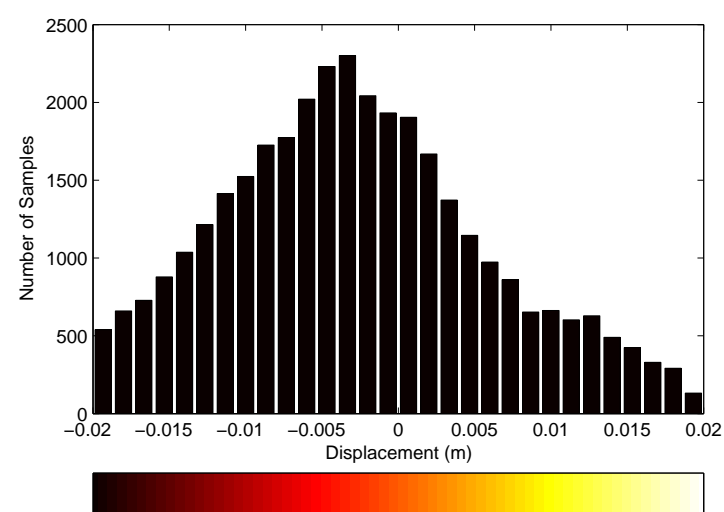

(d) Histogram of displacement values and colour scale

Figure 7. Reconstruction from Multiple All-Around Views 

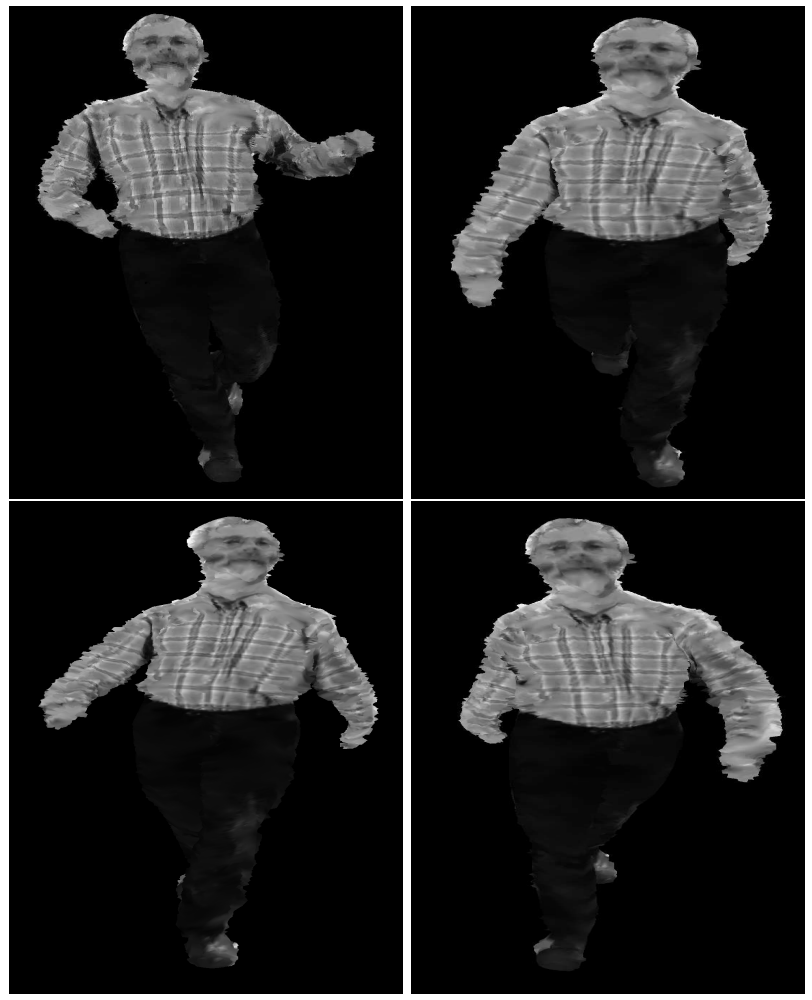

Figure 8. Animation sequence of displacement mapped model 